\title{
Characterization of Migration and Induced Resistance of Rhizobium Vitis Strain ARK-1, a Biological Control Agent Against Grapevine Crown Gall Disease
}

Akira Kawaguchi ( $\sim$ kawaguchia240@affrc.go.jp )

National Agriculture and Food Research Organization

Yoshiteru Noutoshi

Okayama University

\section{Research Article}

Keywords: Rhizobium vitis, grapevine crown gall, biological control

Posted Date: November 24th, 2020

DOI: https://doi.org/10.21203/rs.3.rs-109272/v1

License: (c) (1) This work is licensed under a Creative Commons Attribution 4.0 International License. Read Full License 


\section{Abstract}

A nonpathogenic strain ARK-1 of Rhizobium vitis has an antagonistic activity toward tumorigenic (Ti) strain of $R$. vitis, a causal agent of grapevine crown gall disease, and works as a biocontrol agent. We have demonstrated that the gall formation was fully suppressed when ARK-1 was co-inoculated with $\mathrm{Ti}$ into the grapevine stem at a 1:1 ratio. For practical use of ARK-1 in agriculture, understanding the temporal dynamics of the bacterial habitat on host plants and the biocontrol property are needed in order to develop proper application methods. Here we demonstrated that the gall incidence by Ti was reduced to about $50 \%$ when ARK-1 was pre-inoculated at both upper and lower positions on the grapevine stem 3 $\mathrm{cm}$ away from the Ti-inoculation point 5 days before. The bacterial cell detection assay in the grapevine tissue revealed that ARK-1 could migrate at least $3 \mathrm{~cm}$ in 5 days. Inoculations of ARK-1 or Ti induced expression of marker genes for defense-related phytohormones such as salicylic acid, jasmonic acid, and ethylene in grapevine within 3 days but they were diminished by 6 days. Inoculation of Ti 5 days after ARK-1 pre-inoculation induced expression of the marker genes except for the LOX-9 gene in a basically similar way to those without the pre-inoculation, suggesting that ARK-1 did not induce typical acquired systemic resistance or induced systemic resistance in grapevine, while the transcript of LOX-9 was detected at 24 and 48 hours after the Ti inoculation when ARK-1 was pre-inoculated, unlike the uninoculated condition. ARK-1 primed the induction of certain defense genes and it may take part in its biocontrol activity.

\section{Introduction}

Grapevine (Vitis vinifera L.) crown gall is caused mainly by Rhizobium vitis (Ti) [syn. Agrobacterium vitis (Ti), A. tumefaciens biovar 3], where "Ti" means "tumor-inducing" or "tumorigenic." In this paper, we follow the nomenclature for Rhizobium species adopted by Young et al. ${ }^{1}$ to avoid confusion. This pathogen enters the grapevine through wounds due to a variety of causes, such as winter injury, mechanical damage, and grafting ${ }^{2}$. R. vitis $(\mathrm{Ti})$ causes crown gall by transferring the T-DNA region of the tumor-inducing bacterial plasmid (Ti-plasmid) to the host cell, which subsequently integrates into the plant host genome ${ }^{3-5}$. The inserted T-DNA contains genes for biosynthesis of plant growth hormones, which induce hyperplastic and hypertrophic cell proliferation that results in galls ${ }^{6-7}$. Invasion of vascular tissue by galls can result in vine death ${ }^{8-9}$.

The history of the search for viable biological control agents for crown gall goes back to the early $1970 s^{10}$. Among potential biological agents against crown gall, two have been extensively studied. Rhizobium rhizogenes (=A. rhizogenes, $A$. radiobacter biovar 2) strain $\mathrm{K} 84$ has been used since the early 1970s. It inhibits the growth of some Rhizobium species and reduces crown gall ${ }^{10-12}$. K84 produces an antimicrobial molecule, agrocin 84 , that is antagonistic to particular strains of Rhizobium ${ }^{13-14}$. K84 is used today for the prevention of crown gall predominantly in stone fruit and apple but also other fruit and nut trees. However, K84 is ineffective against crown gall disease in grapevine caused by $R$. vitis ${ }^{7,15-16}$. 
Although several laboratories have attempted to develop biological measures to control grapevine crown gall, there are no successful agents to control this disease, especially under field conditions ${ }^{17-20}$. Previously, we reported that a nonpathogenic $R$. vitis strain, VAR03-1, which was isolated from grapevine nursery stock in Japan, inhibited tumor formation in grapevine, rose, tomato, sunflower, and apple $15,21-24$. Moreover, we identified a nonpathogenic $R$. vitis strain ARK-1 as a new antagonistic $\operatorname{strain}^{16}$. ARK-1 neither carries the Ti-plasmid nor causes disease symptoms ${ }^{16}$. ARK-1 is endophytic in grapevine and induces no necrosis in the host plant. It provided better control against grapevine crown gall than VAR031 in field trials ${ }^{25}$. ARK-1 is also effective at controlling crown gall in other plant species such as tomato, pear, peach, apple, and rose ${ }^{26}$. Suppression of gall formation by ARK-1 was observed when it was coinoculated with Ti into a grapevine stem. The culture filtrate (CF) of ARK-1 (without cells) or heat-killed culture was not able to inhibit tumor formation on grapevine ${ }^{16}$. These results indicate that ARK-1 must be alive to effectively antagonize gall formation by $\mathrm{Ti}^{27}$. ARK- 1 has antibiotic activity against $\mathrm{Ti}$ strains of Rhizobium spp. and this direct antagonism would be an important mechanism for its biocontrol activity $^{28}$.

Pre-treatment of grapevine roots with ARK-1 cell suspension before planting in Ti-contaminated soil also effectively suppressed gall formation in roots ${ }^{16,25}$. ARK-1 slowed down the population growth of $\mathrm{Ti}$ at inoculation sites ${ }^{28,29}$ and suppressed the expression of virulence (vir) genes (virA, virD2, virD3, virE2, and virG on Ti-plasmid) that are required for plant cell transformation by T-DNA ${ }^{29,30}$. To develop a practical application method of ARK-1 in agriculture, an understanding of colonization dynamics on host plants is required. It is known that $\mathrm{Ti}$ strains can move inside grapevines through the vascular system and spread widely, thereby developing galls not only on roots but also shoots ${ }^{7,31-32}$. ARK-1 is also expected to migrate through grapevine tissues but it has not yet been characterized.

Plants protect themselves from microorganisms by inducing a coordinated defense system triggered by immunity-related phytohormones such as salicylic acid (SA), jasmonic acid (JA), and ethylene $(E T)^{33}$. To counteract biotrophic pathogens, the SA-dependent signaling pathway plays an important role. In contrast, plants induce defenses against necrotrophic pathogens, insect herbivores, and wounding using JA and ET. In both cases, whole plant tissues become sensitive after an initial attack and respond quickly and strongly to secondary challenges to confer a broad-spectrum resistance. These phenomena are known as systemic acquired resistance (SAR) and wound-induced systemic resistance (WSR), respectively ${ }^{34-36}$. Fluorescent pseudomonads, which are known as plant growth-promoting rhizobacteria (PGPR), enhance plant immunity in above-ground parts by a mechanism called induced systemic resistance (ISR ${ }^{37}$, and this is dependent on JA and ET but not SA ${ }^{38}$. Interestingly, ARK-1 also suppressed tumor formation in grapevine shoots when it was co-inoculated with a Ti strain whose growth was not inhibited by ARK-1 in the antibiosis assay ${ }^{28}$. Based on this result, we expect that ARK-1 has another mechanism other than antibiosis, such as immune priming or conflict of habitat, for the suppression of crown gall. However, it remains unclear. 
In this study, we verified that ARK-1 can move along the grapevine stem and suppress gall formation by $\mathrm{Ti}$ at places distant from the inoculation site. ARK-1 reached positions $3 \mathrm{~cm}$ away within 5 days and reduced disease incidence by $\mathrm{Ti}$ to about $50 \%$ at that position. The inoculation of ARK-1 upregulated defense-related genes $24-72$ hours post-inoculation (hpi) in the same manner as $\mathrm{Ti}$ in grapevine, but the upregulation was transient. Pre-inoculation of ARK-1 did not enhance expression of the defense-related genes tested upon $\mathrm{Ti}$ inoculation except for $L O X-9$, suggesting the possibility of an atypical priming effect of ARK-1 on particular genes.

\section{Materials And Methods}

\section{Bacterial strains}

The bacterial strains used in this study are listed in Table S1. Potato sucrose agar (PSA) medium was used for culture of the bacteria. A streptomycin (St)- and copper sulfate $\left(\mathrm{CuSO}_{4}\right)$-resistant ARK-1 mutant (ARK-1 sc) was selected using PSA medium containing $500 \mu \mathrm{g} / \mathrm{mL} \mathrm{St} \mathrm{and} 250 \mu \mathrm{g} / \mathrm{mL} \mathrm{CuSO}_{4}{ }^{25}$. A nalidixic acid (nal)-resistant VAT03-9 (Ti) mutant (VAT03-9n) was obtained using PSA medium supplemented with $50 \mu \mathrm{g} / \mathrm{mL} \mathrm{nal}{ }^{29}$. The growth rates of these mutants in PSA medium were comparable to that of the wild-type ${ }^{29}$.

\section{Gall inhibition assay in grapevine seedlings}

Grapevine seedlings (Vitis vinifera cv. 'Neo Muscat') were grown from seed. One-year-old grapevine shoots were inoculated using previously established methods (Kawaguchi et al. 2005, 2007). For the preparation of cell suspensions of ARK-1 and Ti, bacterial cells collected from 48-h-old cultures on PSA slant medium were suspended in distilled water and adjusted to OD600 $=0.1$ (ca. $10^{8} \mathrm{cells} / \mathrm{mL}$ ) after washing once. A $5-\mu \mathrm{L}$ solution (containing approximately $5 \times 10^{5}$ cells) of them was dropped onto needleprick wounds on the stems of grapevine seedlings.

In this study, four inoculation patterns (treatments) with three different inoculation points were used (Table 1, Fig. S1). Each grapevine seedling (at one plant per pot) had three inoculation sites (upper, middle, and lower) with approximately $3.0 \mathrm{~cm}$ spacing. In treatment (i), Ti was inoculated into the middle point, and at the same time sterile distilled water was inoculated into the upper and lower points. In treatment (ii), Ti was inoculated into the middle point, and at the same time ARK-1 was inoculated into the upper and lower points. In treatment (iii), sterile distilled water was pre-inoculated into the upper and lower points, and 5 days later, Ti was inoculated into the middle point. In treatment (iv), ARK- 1 was preinoculated into the upper and lower points and, inoculated into the middle point after 5 days. Ten plants were used for each treatment. The experiments were performed independently 3 times. Grapevine seedlings were grown in a greenhouse at $20-35^{\circ} \mathrm{C}$ with natural sunlight (photoperiod: 12 to $14 \mathrm{~h}$ light: 10 to $12 \mathrm{~h}$ dark). The inoculated seedlings were kept in the same environment for 5 months, and gall formation rates and gall sizes (diameter) $(\mathrm{mm})$ (the widest point measured with a digital caliper) were recorded. 
To monitor the populations of ARK-1 and Ti, treatments (i), (ii), (iii), and (iv) were performed by using the antibiotic-resistant mutants ARK-1 sc and VAT03-9n (Fig. S1, Table 1). In the ARK-1 migration assay in grapevine seedlings, ARK- $1 \mathrm{sc}$ was inoculated into the middle position. To determine the bacterial population, stem parts (ca. $0.2 \mathrm{~g}$ fresh weight per plant, 1 sample per plant) were collected from five plants (i.e., $n=5$ ). Then each sample was scrubbed by hand under running tap water for $10 \mathrm{~s}$, and the water drops were wiped off with paper towels. The samples were crushed in $1 \mathrm{~mL}$ of sterile distilled water using an autoclaved mortar and pestle. Ten-fold serial dilutions $(100 \mu \mathrm{L})$ of each sample were prepared and spread on PSA media containing antibiotics. The plates were incubated at $25^{\circ} \mathrm{C}$ for $6 \mathrm{~d}$, and the numbers of colony-forming units (CFU) were counted.

\section{Gene expression analysis}

For the gene induction assay shown in Fig. 3 , ARK-1, Ti, or water (ca. $0.2 \mathrm{~g}$ ) was inoculated into the middle position of seedling stems and shoot parts $5 \mathrm{~mm}$ away from the inoculation site and collected from three plants at 12, 24, 48, and $72 \mathrm{hpi}$. For the priming assay shown in Fig. 4, each bacterium was inoculated as treatment (iii) or (iv) (Fig. S1, Table 1). Shoot samples were collected as mentioned above at 24, 48, and $72 \mathrm{hpi}$. Total RNA was extracted from the frozen samples using the NucleoSpin RNA (Macherey-Nagel, Düren, Germany). To remove the genomic DNA in the extracted RNA and synthesize cDNA from $1.0 \mu \mathrm{g}$ of total RNA, we used ReverTra Ace qPCR RT Master Mix with gDNA Remover (Toyobo, Osaka, Japan). Quantitative real-time polymerase chain reaction (qPCR) was carried out using Brilliant III Ultra-Fast SYBR Green QPCR Master Mix With Low ROX (Agilent, Santa Clara, CA, USA) and an AriaMx Real-Time PCR System (Agilent) with specific primers for pathogenesis-related genes ( $P R-1$ and $P R-4)$, plant defensin 1.2 (PDF1.2), lipooxygenase 9 (LOX-9), and ethylene-responsive transcription factor ERF003 (ERF) ${ }^{39-42}$ (Table S2). PR genes were used as a marker of SA signaling. and PDF1.2 and LOX-9 were used as markers of jasmonic acid (JA) signaling. ERF was used as a marker of ethylene (ET) signaling. The PCR conditions were $95^{\circ} \mathrm{C}$ for $10 \mathrm{~min}$, followed by 50 cycles of $95^{\circ} \mathrm{C}$ for $15 \mathrm{~s}$ and $60^{\circ} \mathrm{C}$ for $1 \mathrm{~min}$. Each sample was examined with three technical replicates, and dissociation curves were analyzed to verify the specificity of each amplification reaction. Cycle threshold values were calculated using AriaMx software version 1.5 (Agilent). The relative expression of each gene was obtained according to the Pfaffl equation ${ }^{43}$ using actin as a reference gene ${ }^{44,45}$. Relative expression rates were calculated in comparison with values of the expression level of each gene at 12 or 24 hpi as 1.0 for Fig. 3 and Fig. 4, respectively. The experiments were performed 3 times for each assay.

\section{Data analysis}

Tukey's honestly significant difference (HSD, a $=0.05)$ test (after analysis of variance, ANOVA) was used to compare the $\mathrm{CFU} / \mathrm{g}$ of plant tissue, relative gene expression, and gall size in the various treatments. Ryan's multiple-comparison $(a=0.05)$ test was used to compare the frequency of tumor formation among 
treatments. All statistical analyses were performed using version 3.6.1 of the R software (http://www.rproject.org/).

\section{Results}

\section{ARK-1 suppressed Ti-induced gall formation in positions distant from the inoculated site}

To evaluate the biocontrol performance of ARK-1 at a position distant from the inoculation site, we inoculated grapevine stem at 3 inoculation sites (upper, middle, and lower) at $3 \mathrm{~cm}$ intervals (Fig. S1). We inoculated cell suspensions of Ti and ARK-1 into grapevine seedling stems with different combinations of timing, and then measured gall incidence (Fig. 1A) and gall size (Fig. 1B) at each point after 5 months. When Ti was inoculated into the middle position and water was inoculated into both upper and lower positions at the same time (treatment (i)) (Fig. S1), galls were formed at all inoculation sites with an incidence of 100\% (Fig. 1A). Each gall at the upper and lower positions was 2.58 and $2.89 \mathrm{~mm}$, respectively, and both sizes were significantly smaller than that at the middle position $(3.83 \mathrm{~mm}$ ) (Figs. $1 \mathrm{~B}$ and $2 \mathrm{~A}$ ). This result indicated that Ti moved $3 \mathrm{~cm}$ and produced galls at the position if it was wounded. Then, we inoculated Ti into the middle position and ARK-1 into both upper and lower positions at the same time (treatment (ii)) (Fig. S1). In this case, galls were formed only at the middle positions with $100 \%$ incidence (Fig. 1A). Their sizes were similar to those in the middle position of treatment (i) (Fig. 1B). In this case, almost no galls were observed at either upper or lower positions (average gall incidence was $3.33 \%$ ) (Figs. 1A and 2B). This meant that ARK-1 suppressed the virulence of $\mathrm{Ti}$ at the upper and lower positions but it had no effect on the distant positions when ARK-1 and Ti were inoculated together. Next, we inoculated water into both upper and lower positions, and Ti was inoculated at the middle position 5 days later (treatment (iii)) (Fig. S1). The result was similar to that of the treatment (i). Finally, ARK-1 was inoculated into both upper and lower positions, and Ti was inoculated into the middle position after 5 days (treatment (iv)) (Fig. S1). The gall formations were suppressed at the upper and lower positions (gall incidences were $3.33 \%$ and $0.00 \%$, respectively) as observed in treatment (ii) (Fig. $1 \mathrm{~A}$ ). Interestingly, the gall incidence at the middle position was $60.00 \%$, which was $40.00 \%$ lower than that in treatment (iii) and its gall sizes were significantly smaller (Figs. 1A and 1B). These results implied that ARK-1 could move at least $3 \mathrm{~cm}$ in 5 days and suppress the virulence of Ti. Note that no galls were found when water alone was inoculated as the negative control (data not shown).

\section{ARK-1 migrated in grapevine seedling stem}

To further confirm the possibility that ARK-1 moves $3 \mathrm{~cm}$ in 5 days in grapevine stems, the population of ARK-1 was measured in the region distant from the inoculation point. For this purpose, the spontaneously emerged antibiotic-resistant ARK-1 mutant (ARK-1sc) was used. ARK-1sc is resistant to both streptomycin and $\mathrm{CuSO}_{4}$. We inoculated a cell suspension of ARK-1sc into the middle position in grapevine seedling stems, and the inoculation and the upper and lower sites $3 \mathrm{~cm}$ away from the inoculation were sampled. The bacterial concentrations in the extracts of these plant tissues were measured by counting colonies grown on a PSA medium containing the antibiotics. At the inoculation point, 5,623,413 CFU/g was 
detected (Table 2). On the other hand, 426,579 and 79,432 CFU/g were detected in the upper and lower inoculation positions, respectively (Table 2 ). They corresponded to $7.5 \%$ and $1.4 \%$ of the population in the inoculated point. This result showed that ARK-1 can migrate at least $3 \mathrm{~cm}$ to both upward and downward directions in 5 days through grapevine stems after inoculation.

\section{Defense-related genes were induced 2-3 days after inoculation of ARK-1 and Ti}

Anand et al. ${ }^{46}$ reported that SA and SAR play major roles in the resistance of Nicotiana benthamiana to $R$. radiobacter $(\mathrm{Ti})(=$ A. tumefaciens $(\mathrm{Ti}))$. Hao et al. ${ }^{47}$ reported that $R$. radiobacter $(\mathrm{Ti})$ induced the $P R-1$ gene in peach. Based on these findings, we speculated that increased defense such as SAR or ISR in host plants is part of the biocontrol activity of ARK-1. To verify this possibility, expressions of defense-related marker genes in grapevine were monitored after the inoculation of ARK-1 or Ti. In this study, we used $P R-1$ and PR-4 for SA, PDF1.2 and LOX-9 for JA, and ERF for ET as marker genes for defense-related phytohormones ${ }^{40-42}$. Each bacterial strain was inoculated into stems of grapevine seedlings and plant tissues $5 \mathrm{~mm}$ from the inoculation points and sampled at 12, 24, 48, and $72 \mathrm{hpi}$. As a result, an increase (6-100 fold) of the expressions of $P R-1, P R-4, L O X-9$, and ERF was detected at $72 \mathrm{hpi}$ upon the inoculation of both bacterial strains compared with the water control (Fig. 3). The expressions of PDF1.2 seemed to be slightly increased from $24 \mathrm{hpi}$, but the increases were small and unclear (Fig. 3). These results indicated that $R$. vitis could upregulate some kinds of defense responses possibly related to $S A$, $\mathrm{JA}$, and ET in the surrounding area of the inoculation site in grapevine at 72 hpi regardless of its virulence.

\section{Pre-inoculation of ARK-1 enhanced LOX-9 gene expression upon Ti inoculation}

We examined whether the pre-inoculation of ARK-1 can make the plants more sensitive to subsequent pathogen infection during defense induction. We inoculated ARK-1 or water into both upper and lower positions on stems of grapevine seedlings as treatment (iv), and Ti was inoculated to the middle position after 5 days. Plant tissues $5 \mathrm{~mm}$ away from the Ti-inoculation point were sampled at 24,48 , and $72 \mathrm{hpi}$ for further analysis of gene expression. $P R-1, P R-4, P D F 1.2$, and $E R F$ were upregulated at $72 \mathrm{hpi}$ in response to the inoculation of $\mathrm{Ti}$ when water was pre-inoculated to the upper and lower positions. This was basically consistent with the results obtained without the pre-inoculation, as demonstrated in Fig. 3 , although PDF1.2 expression was relatively higher (Fig. 4). In contrast, the expression levels of $P R-4$ and ERF at $72 \mathrm{hpi}$ and PDF1.2 at 36 and $72 \mathrm{hpi}$ after the Ti inoculation in the ARK-1-pretreated condition were decreased compared with those of the water-pretreated experiment. These results showed that ARK-1 seemed not to induce typical SAR or ISR, unlike the previously characterized bacterial biocontrol agents such as Pseudomonas fluorescens. However, LOX-9 gene was induced by Ti at 24 and 48 hpi only when ARK-1 had been pre-inoculated into the distant position 5 days before. The expression of a particular range of defense genes may be primed by ARK-1. 


\section{Discussion}

Our inoculation system with separate inoculation sites in the grapevine shoot revealed the migration capacities of ARK-1 and Ti. Ti could move at least $3 \mathrm{~cm}$ within 5 days and produced galls at that position if the site had been injured. Because the recognition of phenolic molecules such as acetosyringone is required for triggering of the virulence program in Rhizobium/Agrobacterium, such compounds should still remain at the wound site even after 5 days. This is basically consistent with the previous report showing that $R$. vitis ( $\mathrm{Ti}$ ) moved to $30 \mathrm{~cm}$ away from the entry point within $24 \mathrm{hpi}^{31}$. On the other hand, $\mathrm{Ti}$ did not produce galls when it translocated to the site where ARK-1 was pre-inoculated. As we have already reported ${ }^{25}$, this result supports the usefulness of the pretreatment method of grapevine roots with the ARK-1 cell suspension before transplantation to control crown gall disease. In this study, the migration of ARK-1 along with the grapevine seedling stem was demonstrated in a manner similar to that of Ti. The ARK-1 cell density at the upper position $3 \mathrm{~cm}$ away from the inoculation point was higher than that of the lower position when ARK-1 had been inoculated into the middle position 5 days before. It may be easier to move upward than downward. ARK-1 translocated at least $3 \mathrm{~cm}$ within 5 days as well as $\mathrm{Ti}$, and the gall incidence dropped by $60 \%$ at the site. This means that ARK-1 could exert its protective effect at the distant position, but it was still insufficient for full suppression. This would be due to the cell density. The ARK-1 populations at both upper and lower positions $3 \mathrm{~cm}$ away from the inoculation site at $5 \mathrm{dpi}$ were 7.5 and $1.4 \%$ of that at the inoculation point, respectively. In other words, even small numbers of ARK-1 could work to a certain extent. We previously demonstrated that ARK-1 was effective in preventing gall formation on grapevine shoots when it was co-inoculated with Ti strains at a 1:1 cell ratio ${ }^{16,27}$. Although the effective concentration of ARK-1 is now being investigated, in the case of $R$. vitis VAR03-1 gall formation was suppressed when it was inoculated with the pathogen at a 1:1 ratio, but its control activity was decreased at 1:3 or 1:9 ratios ${ }^{15}$. Given the results obtained so far, in terms of practical usage of ARK1 as a biocontrol agent for the management of crown gall disease in grapevine, we should allow a sufficient period of time after pretreatment of grapevine shoots with ARK-1 in a non-contaminated place before transplanting them to the field to wait for the bacterial density to be high enough. In addition to this pre-inoculation method, our results also showed the potential of ARK-1 treatment of growing grapevine trees in the field. Although there is currently no practical way to save grapevine trees from crown gall disease in a pathogen-contaminated field, inoculated ARK-1 may spread throughout whole plants gradually and protect them. Further investigation is needed to clarify if ARK-1 can translocate over long distances and suppress Ti-induced galls at places distant from the entry point in mature grapevine trees.

In this study, we tried to confirm the possibility of the involvement of increased defense in the biocontrol activity of ARK-1. The selected defense marker genes, $P R-1, P R-4, L O X-9, P D F 1.2$, and ERF, for SA, JA, and ET were increased over 3 days after the inoculation of ARK-1 or Ti near the inoculation point (Fig. 3 ). This result indicates that grapevine induced defense responses to these bacteria. However, such defense responses were not detected at the ARK-1 inoculation point on the sixth day (Fig. 4). Therefore, the response to these bacteria would be diminished after 6 days. These results indicate that the direct 
induction of defense responses in host plants would not be the major factor in the ARK-1 biocontrol activity. The induction patterns of defense genes, $P R-1, P R-4, E F F$, and $P D F 1.2$, upon Ti inoculation at the ARK-1 pre-inoculation points were similar to those to ARK-1 or Ti inoculation without pre-inoculation (Figs. 3 and 4). This also supports the idea that the defense responses have already decreased by the initial inoculation. The expressions of these genes were not accelerated and enhanced, suggesting that at least typical SAR and ISR may not be mounted by ARK-1. However, an unexpected response was detected in LOX-9. The LOX-9 gene was induced at 24 and $48 \mathrm{~h}$ after Ti inoculation only in the ARK-1 pre-treated plants. This result suggested that ARK-1 might facilitate the expression of a particular gene(s). LOX is known to be involved in the production of oxidized fatty acids in plants that can be the precursor of antibiotic compounds or signal molecules such as oxylipins ${ }^{41,48,49}$. The activation of systemic resistance by nonpathogenic rhizobacteria, Bacillus spp. and Pseudomonas putida, has been associated with the induction of LOX activity in bean and tomato ${ }^{50}$. Although the biological role of $L O X-9$ gene used in this study is unknown, it may somehow contribute to the ARK-1 biocontrol activity. Further transcriptome analysis could help clarify the role of this unique priming activity in its suppressive activity for crown gall disease.

\section{Declarations}

\section{Acknowledgments}

This research was supported by KAKENHI Grant $17 \mathrm{H} 03778$ and 20K20572 from the Ministry of Education, Culture, Sports, Science and Technology of Japan to A. Kawaguchi and Y. Noutoshi. We are grateful to Dr. K. Inoue (Research Institute for Agriculture, Okayama Prefectural Technology Center for Agriculture, Forestry and Fisheries) who gave us much useful advice.

\section{Compliance with ethical standards}

\section{Conflict of interest}

The authors declare no competing financial interests.

\section{Ethical standards}

This article does not contain any studies with human participants or animals performed by the authors.

\section{References}

1. Young, J.M., Kuykendall, L.D., Martínez-Romero, E., Kerr, A. \& Sawada, H. A revision of Rhizobium Frank 1889, with an emended description of the genus, and the inclusion of all species of Agrobacterium Conn 1942 and Allorhizobium undicola de Lajudie et al. 1998 as new combinations: Rhizobium radiobacter, rhizogenes, $R$. rubi, $R$. undicola and $R$. vitis. Int. J. Syst. Evol. Microbiol. 51:89-103 (2001). 
2. Burr, T.J. \& Otten, L. Crown gall of grape: Biology and disease management. Rev. Phytopathol. 37:53-80 (1999).

3. Chilton, M.D. et al. Stable incorporation of plasmid DNA into higher plant cells: the molecular basis of crown gall tumorigenesis. Cell 11:263-271 (1977).

4. Pitzschke, A., \& Hirt, H. New insights into an old story: Agrobacterium-induced tumour formation in plants by plant transformation. EMBO J. 29:1021-1032 (2010). https://doi.org/10.1038/emboj.2010.8

5. Gelvin, S.B. Traversing the cell: Agrobacterium T-DNA's journey to the host genome. Plant Sci. 3:52 (2012).

6. Morris, R. Genes specifying auxin and cytokinin biosynthesis in phytopathogens. Rev. Plant Physiol. 37:509-538 (1986).

7. Burr, T.J., Bazzi, C., Süle, S. \& Otten, L. Crown gall of grape: Biology of Agrobacterium vitis and the development of disease control strategies. Plant Dis. 82:1288-1297 (1998).

8. Wächter, R. et al. Vascularization, high-volume solution flow, and localized roles for enzymes of sucrose metabolism during tumorigenesis by Agrobacterium tumefaciens. Plant Physiol. 133:10241037 (2003).

9. J. \& Deeken, R. Plant responses to Agrobacterium tumefaciens and crown gall development. Front. Plant Sci. 5:155 (2014).

10. New, P.B. \& Kerr, A. Biological control of grown gall: Field measurements and glasshouse experiments. Appl. Bacteriol. 35:279-287 (1972).

11. Htay, K. \& Kerr, A. Biological control of crown gall: Seed and root inoculation. Appl. Bacteriol. 37:525530 (1974).

12. Kerr, A. \& Htay, K. Biological control of crown gall through bacteriocin production. Plant Pathol. 4:3744 (1974).

13. Kerr, A. Biological control of crown gall through production of agrocin 84. Plant Dis. 64:24-30 (1980).

14. Reader, J.S. et al. Virology: Major biocontrol of plant tumors targets tRNA synthetase. Science 309:1533 (2005).

15. Kawaguchi, A., Inoue, K. \& Nasu, H. Biological control of grapevine crown gall by nonpathogenic Agrobacterium vitis strain VAR03-1. Gen. Plant Pathol. 73:133-138 (2007).

16. Kawaguchi, A. \& Inoue, K. New antagonistic strains of non-pathogenic Agrobacterium vitis to control grapevine crown gall. Phytopathol. 160:509-518 (2012).

17. Staphorst, J.L., van Zyl, F.G.H., Strijdom, B.W. \& Groenewold, Z.E. Agrocin-producing pathogenic and nonpathogenic biotype-3 strains of Agrobacterium tumefaciens active against biotype-3 pathogens. Microbiol. 12:45-52 (1985).

18. Burr, T.J. \& Reid, C.L. Biological control of grape crown gall with non-tumorigenic Agrobacterium vitis strain F2/5. J Enol. Vitic. 45:213-219 (1994). 
19. Wang, H.M., Wang, H.X., Ng, T.B. \& Li. J. Y. Purification and characterization of an antibacterial compound produced by Agrobacterium vitis strain E26 with activity against tumefaciens. Plant Pathol. 52:134-143 (2003).

20. Chen, F., Guo, Y.B, Wang, J.H. Li, J.Y. \& Wang, H.M. Biological control of grape crown gall by Rahnella aquatilis Plant Dis. 91:957-963 (2007).

21. Kawaguchi, A., Inoue K. \& Nasu H. Inhibition of crown gall formation by Agrobacterium radiobacter biovar 3 strains isolated from grapevine. Gen. Plant Pathol. 71:422-430 (2005).

22. Kawaguchi, A., Inoue K. \& Ichinose Y. Biological control of crown gall of grapevine, rose, and tomato by nonpathogenic Agrobacterium vitis strain VAR03-1. Phytopathology 98:1218-1225 (2008).

23. Kawaguchi, A., Kondo, K. \& K. Inoue, K. Biological control of apple crown gall by nonpathogenic Rhizobium vitis strain VAR03-1. Gen. Plant Pathol. 78:287-293 (2012).

24. Saito, K. et al. Characterization of the suppressive effects of the biological control strain VAR03-1 of Rhizobium vitis on the virulence of tumorigenic vitis. J. Gen. Plant Pathol. 84:58-64 (2018).

25. Kawaguchi, A. Biological control of crown gall on grapevine and root colonization by nonpathogenic Rhizobium vitis strain ARK-1. Microbes Environ. 28:306-311 (2013).

26. Kawaguchi, A., Inoue, K. \& Tanina, K. Evaluation of the nonpathogenic Agrobacterium vitis strain ARK-1 for crown gall control in diverse plant species. Plant Dis. 99:409-414 (2015).

27. Kawaguchi, A., Inoue, K., Tanina, K \& Nita, M. Biological control for grapevine crown gall using nonpathogenic Rhizobium vitis strain ARK-1. Japan Acad. Ser. B. Phys. Biol. Sci. 93:547-560 (2017).

28. Kawaguchi, A. Reduction in pathogen populations at grapevine wound sites is associated with the mechanism underlying the biological control of crown gall by Rhizobium vitis strain ARK-1. Microbes Environ. 29:296-302 (2014).

29. Kawaguchi, A. Biological control agent Agrobacterium vitis strain ARK-1 suppresses expression of the virD2 and virE2 genes in tumorigenic vitis. Eur. J. Plant Pathol. 143:789-799 (2015).

30. Kawaguchi, A., Nita, M., Ishii, T., Watanabe, M. \& Noutoshi, Y. Biological control agent Rhizobium (=Agrobacterium) vitis strain ARK-1 suppresses expression of the essential and non-essential vir genes of tumorigenic vitis. BMC Res. Notes 12:1-6 (2019).

31. Tarbah, F. \& Goodman, R.N. Systematic spread of Agrobacterium tumefaciens biover 3 in vascular system of grapes. Phytopathology 77:915-920 (1987).

32. Johnson, K.L., Cronin, H., Reid, C.L. \& Burr, T.J. Distribution of Agrobacterium vitis in grapevines and its relevance to pathogen elimination. Plant Dis. 100:791-796 (2016).

33. Thomma, B.P. et al. Separate jasmonate-dependent and salicylate- dependent defense response pathways in Arabidopsis are essential for resistance to distinct microbial pathogens. Natl. Acad. Sci. USA 95:15107-15111 (1998).

34. Dong, X. NPR1, all things considered. Opin. Plant Biol. 7:547-552 (2004).

35. Hilleary, R. \& Gilroy, S. Systemic signaling in response to wounding and pathogens. Opin. Plant Biol. 43:57-62 (2018). 
36. Goellner, K. \& Conrath, U. Priming: it's all the world to induced disease resistance. J. Plant Pathol. 121:233-242 (2008).

37. Pieterse, C.M.J. et al. Rhizobacteria-mediated induced systemic resistance (ISR) in Arabidopsis requires sensitivity to jasmonate and ethylene but is not accompanied by an increase in their production. Mol. Plant Pathol. 57:123-134 (2000).

38. Pieterse, C.M.J., Leon-Reyes, A., Van der Ent, S. \& Van Wees, S.C.M. Networking by small-molecule hormones in plant immunity. Chem Biol. 5:308-316 (2009).

39. Kortekamp, A. Expression analysis of defense-related genes in grapevine leaves after inoculation with a host and a non-host pathogen. Plant Physiol. Biochem. 44:58-67 (2006).

40. Le Henanff, G., Heitz, T., Mestre, P., Mutterer, J., Walter, B. \& Chong, J. Characterization of Vitis vinifera NPR1 homologs involved in the regulation of pathogenesis-related gene expression. BMC Plant Biol. 9:54 (2009).

41. Perazzolli, M., Roatti, B., Bozza, E. \& Pertot, I. Trichoderma harzianum T39 induces resistance against downy mildew by priming for defense without costs for grapevine. Contro/ 58:74-82 (2011).

42. Roatti, B., Perazzolli, M., Gessler, C. \& Pertot, I. Abiotic stresses affect Trichoderma harzianum T39induced resistance to downy mildew in grapevine. Phytopathology 103:1227-1234 (2013).

43. Pfaffl, M.W., Horgan, G.W. \& Dempfle, L. Relative expression software tool (REST) for group-wise comparison and statistical analysis of relative expression results in real-time PCR. Nucleic Acids Res. 30: e36 (2002).

44. Gutha, L.R., Casassa, L.F., Harbertson, J.F. \& Naidu, R.A. Modulation of flavonoid biosynthetic pathway genes and anthocyanins due to virus infection in grapevine (Vitis vinifera) leaves. BMC Plant Biol. 10:187 (2010).

45. Perazzolli, M., et al. Downy mildew resistance induced by Trichoderma harzianum T39 in susceptible grapevines partially mimics transcriptional changes of resistant genotypes. BMC Genomics 13:660 (2012).

46. Anand, A. et al. Salicylic acid and systemic acquired resistance play a role in attenuating crown gall disease caused by Agrobacterium tumefaciens. Plant Physiol. 146:703-715 (2008).

47. Hao, F. et al. Systemic acquired resistance induced by Agrobacterium tumefaciens in peach and differential expression of PR1 Hortscience 50:666-672 (2015).

48. Hamberg, M., Sanz, A., Rodriguez, M. J., Calvo, A. P. \& Castresana, C. Activation of the fatty acid adioxygenase pathway during bacterial infection of tobacco leaves. Bio. Chem. 278:51796-51805 (2003).

49. Vellosillo, T., Vicente, J., Kulasekaran, S., Hamberg, M. \&Castresana, C. Emerging complexity in reactive oxygen species production and signaling during the response of plants to pathogens. Plant Physiol. 154:444-448 (2010).

50. Akram, A., Ongena, M., Duby, F., Dommes, J. \& Thonart, P. (2008) Systemic resistance and lipoxygenase-related defense response induced in tomato by Pseudomonas putida strain BTP1. BMC Plant Biol. 8:113 (2008). 


\section{Tables}

Table 1. Summary of inoculation method (treatments) used in this study

\begin{tabular}{|lllll|}
\hline \multicolumn{5}{|c|}{ Treatments } \\
\hline Inoculation positions on grapevine seedling stem & (i) & (ii) & (iii) & (iv) \\
\hline Upper & Water & ARK-1 & Water & ARK-1 \\
\hline Middle & Ti & Ti & Ti at 5 dpi & Ti at 5 dpi \\
\hline Lower & Water & ARK-1 & Water & ARK-1 \\
\hline
\end{tabular}

Rhizobium vitis strain VAR03-9 was used as Ti. In treatments (i) and (ii), all positions were inoculated at the same time. In treatments (iii) and (iv), Ti was inoculated 5 days post-inoculation (dpi) of water or ARK1 at both upper and lower positions. For population assays, the antibiotic-resistant mutants ARK-1sc and VAT03-9n were used.

Table 2. Population of ARK-1sc in regions distant from the inoculated or un-inoculated point of grapevine seedling stem

\begin{tabular}{|c|c|c|c|}
\hline \multirow{3}{*}{ Inoculant } & \multicolumn{3}{|c|}{ Sampled positions in grapevine seedling stem } \\
\hline & Upper & Middle & Lower \\
\hline & & \Inoculated $\rrbracket$ & \\
\hline ARK-1sc & $5.63 \pm 0.18^{a}$ & $6.75 \pm 0.09^{b}$ & $4.90 \pm 0.14^{c}$ \\
\hline Water & N.D. & N.D. & N.D. \\
\hline
\end{tabular}

ARK-1 sc or water was inoculated at the middle position of grapevine seedling stems, and bacterial populations at each position were measured after 5 days. Data represented as mean $\left(\log _{10} \mathrm{CFU} / \mathrm{g}\right.$ (plant tissue) $) \pm$ SEM $(n=5)$. Different letters represent significant differences according to Tukey's HSD $(P \leq$ 0.05). N.D.; not detected.

\section{Figures}




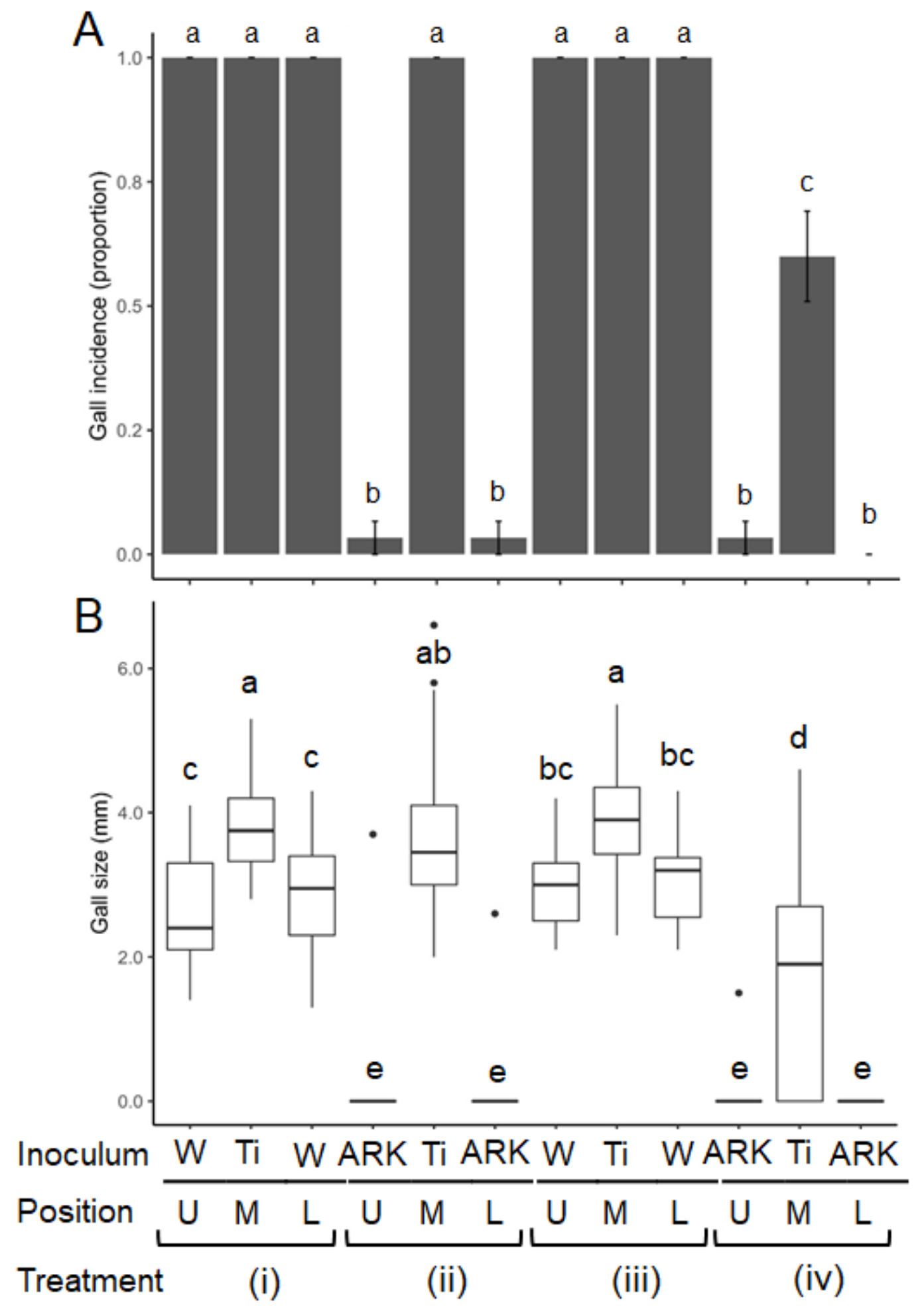

Figure 1

The protective effect of ARK-1 at a distant position. The gall incidence (A) and gall diameter (B) in grapevine stems were measured by 4 different inoculation methods with 3 inoculation positions $3 \mathrm{~cm}$ apart. In treatments (i) and (ii), sterile distilled water or ARK-1 was inoculated into both upper and lower positions and Ti was inoculated into the middle position. In treatments (iii) and (iv), sterile distilled water or ARK-1 was inoculated into both upper and lower positions and Ti was inoculated into the middle 
position after 5 days. Gall formations were evaluated at 5 months after Ti inoculation. (A) Data are represented as means of gall incidence (proportion) with SEM $(n=30)$. Different letters represent significant differences according to Ryan's multiple-comparison test $(P \leq 0.05)$. (B) Distributions of gall sizes are represented by whisker plots $(n=30)$. The horizontal centerline is the median, and the upper and lower parts of the box represent the 75th and 25th percentiles. The points outside the box are outliers. Different letters represent significant differences according to Tukey's HSD $(P \leq 0.05)$. Inoculation sites that did not form galls were given a gall size value of $0.00 \mathrm{~mm}$. W, water. $U$, upper. $M$, middle. L, lower.

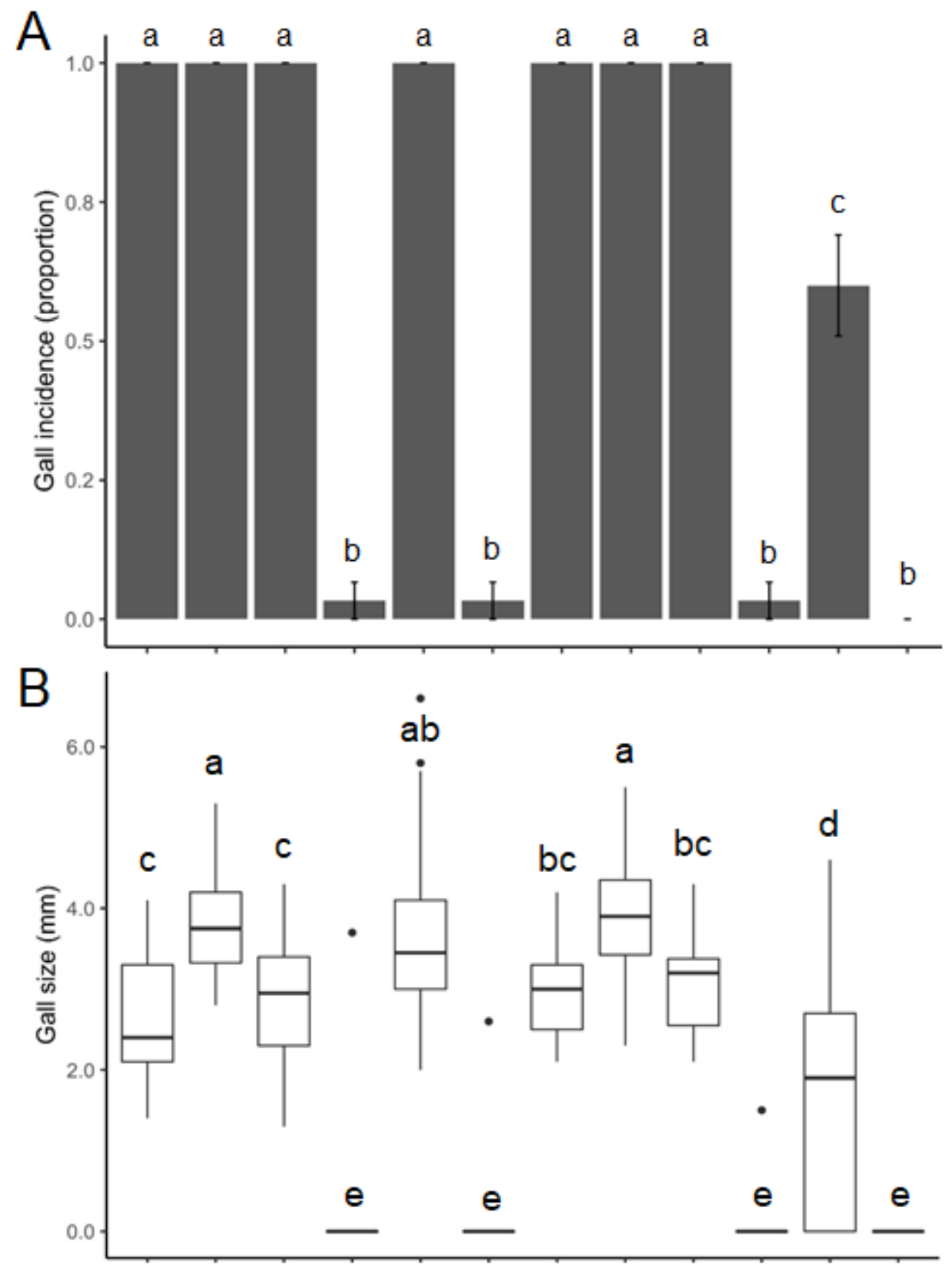

Inoculum $\mathrm{W}$ Ti W ARK Ti ARK W Ti W ARK Ti ARK

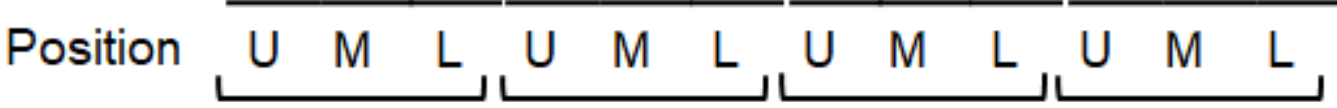

Treatment

(i)

(ii)

(iii)

(iv) 


\section{Figure 1}

The protective effect of ARK-1 at a distant position. The gall incidence (A) and gall diameter (B) in grapevine stems were measured by 4 different inoculation methods with 3 inoculation positions $3 \mathrm{~cm}$ apart. In treatments (i) and (ii), sterile distilled water or ARK-1 was inoculated into both upper and lower positions and Ti was inoculated into the middle position. In treatments (iii) and (iv), sterile distilled water or ARK-1 was inoculated into both upper and lower positions and Ti was inoculated into the middle position after 5 days. Gall formations were evaluated at 5 months after Ti inoculation. (A) Data are represented as means of gall incidence (proportion) with SEM $(n=30)$. Different letters represent significant differences according to Ryan's multiple-comparison test $(P \leq 0.05)$. (B) Distributions of gall sizes are represented by whisker plots $(n=30)$. The horizontal centerline is the median, and the upper and lower parts of the box represent the 75th and 25th percentiles. The points outside the box are outliers. Different letters represent significant differences according to Tukey's HSD ( $\leq \leq 0.05)$. Inoculation sites that did not form galls were given a gall size value of $0.00 \mathrm{~mm}$. W, water. $U$, upper. $\mathrm{M}$, middle. L, lower.
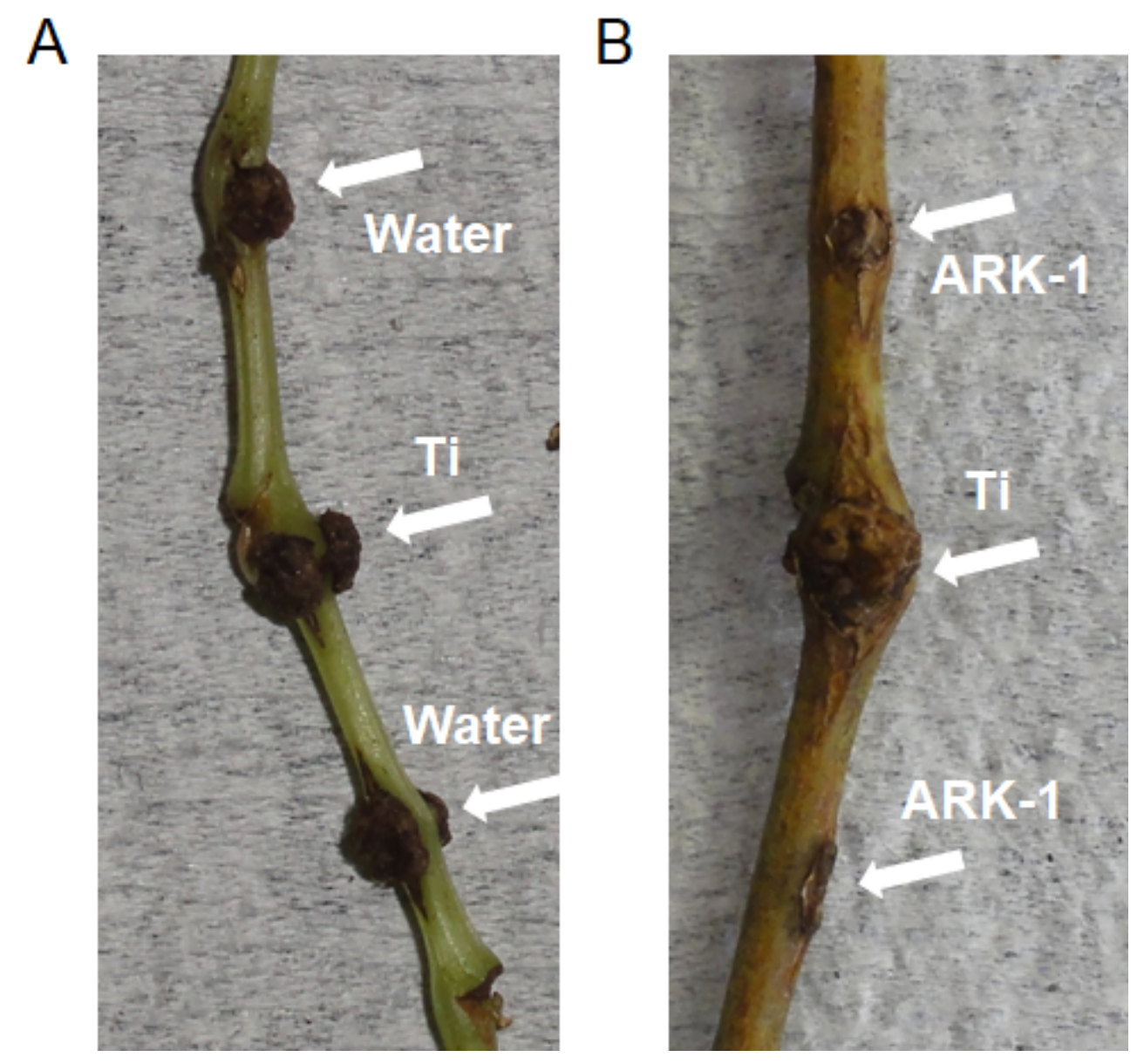

\section{Figure 2}

Photographs of the grapevine shoot 5 months after inoculation by two different methods: treatment (i) (sterile distilled water was inoculated into both upper and lower positions and Ti was inoculated into the middle position) (A) and treatment (ii) (ARK-1 was inoculated into both upper and lower positions and Ti was inoculated into the middle position) (B). Arrows indicate inoculation sites. 

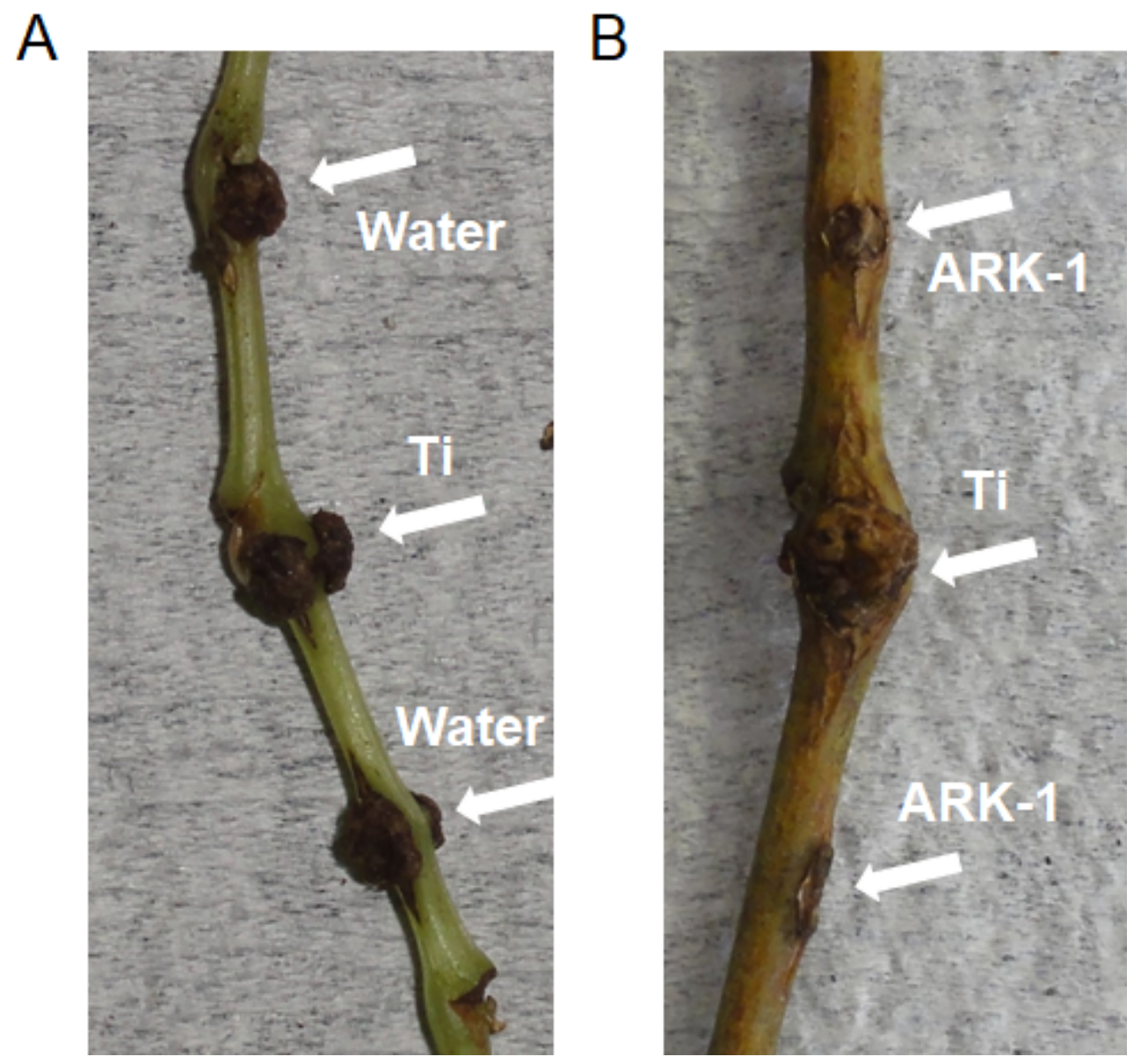

\section{Figure 2}

Photographs of the grapevine shoot 5 months after inoculation by two different methods: treatment (i) (sterile distilled water was inoculated into both upper and lower positions and Ti was inoculated into the middle position) (A) and treatment (ii) (ARK-1 was inoculated into both upper and lower positions and $\mathrm{Ti}$ was inoculated into the middle position) (B). Arrows indicate inoculation sites. 


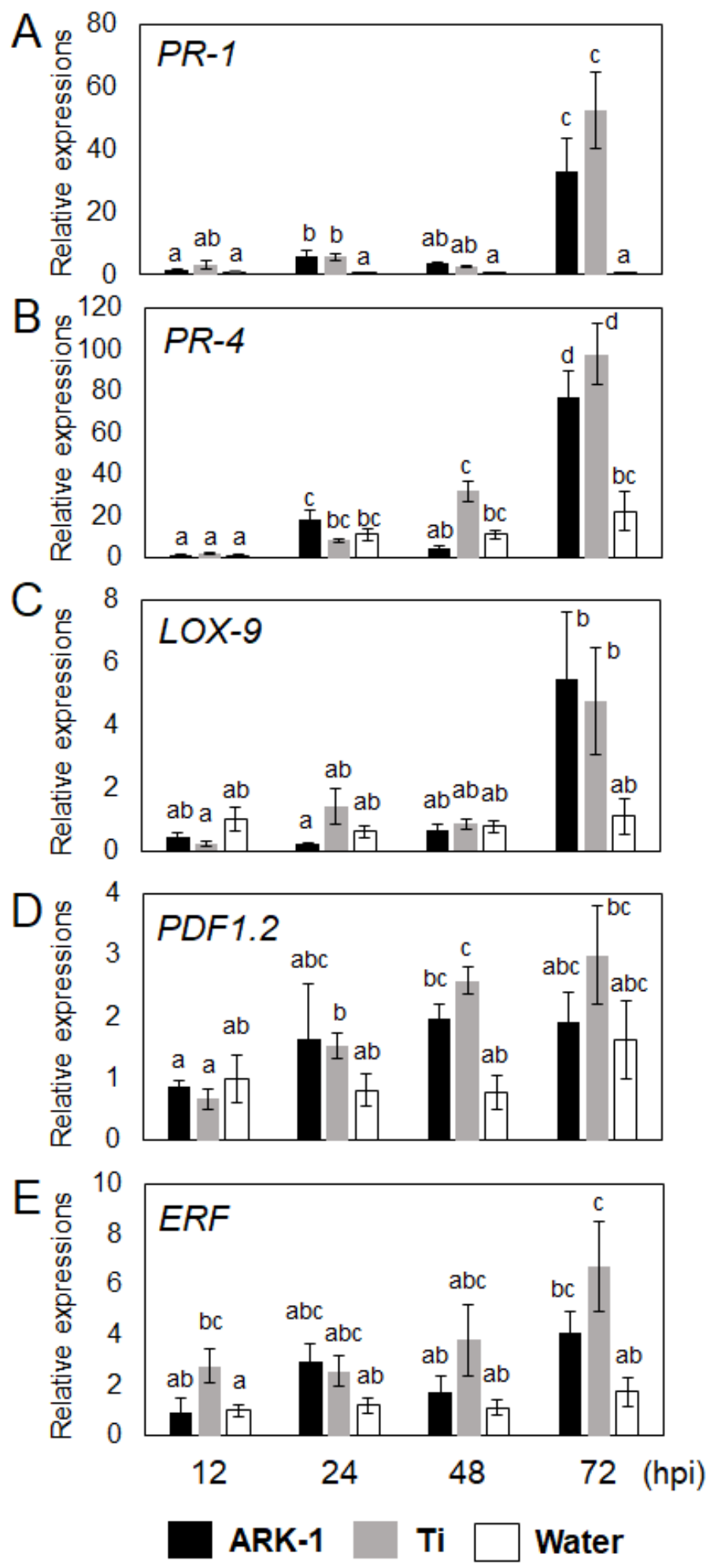

Figure 3

Induction of defense-related genes by ARK- 1 and Ti during 3 days post-inoculation in grapevine seedlings. ARK-1, Ti, or sterile distilled water was inoculated into the middle position, and tissues $5 \mathrm{~mm}$ away from the inoculation sites were collected at the indicated time points. The relative expression rates of defense-related genes PR-1 (A), PR-4 (B), LOX-9 (C), PDF1.2 (D), and ERF (E) were measured using reverse transcriptase quantitative polymerase chain reaction (RT-qPCR) with specific primers. Data are 
means $\pm S E M$ of relative expression rates to each value with water at 12 hours post-inoculation (hpi), which had a value of $1.0(n=3)$. Different letters represent significant differences according to Tukey's HSD $(P \leq 0.05)$. The experiments were repeated 3 times with similar results.

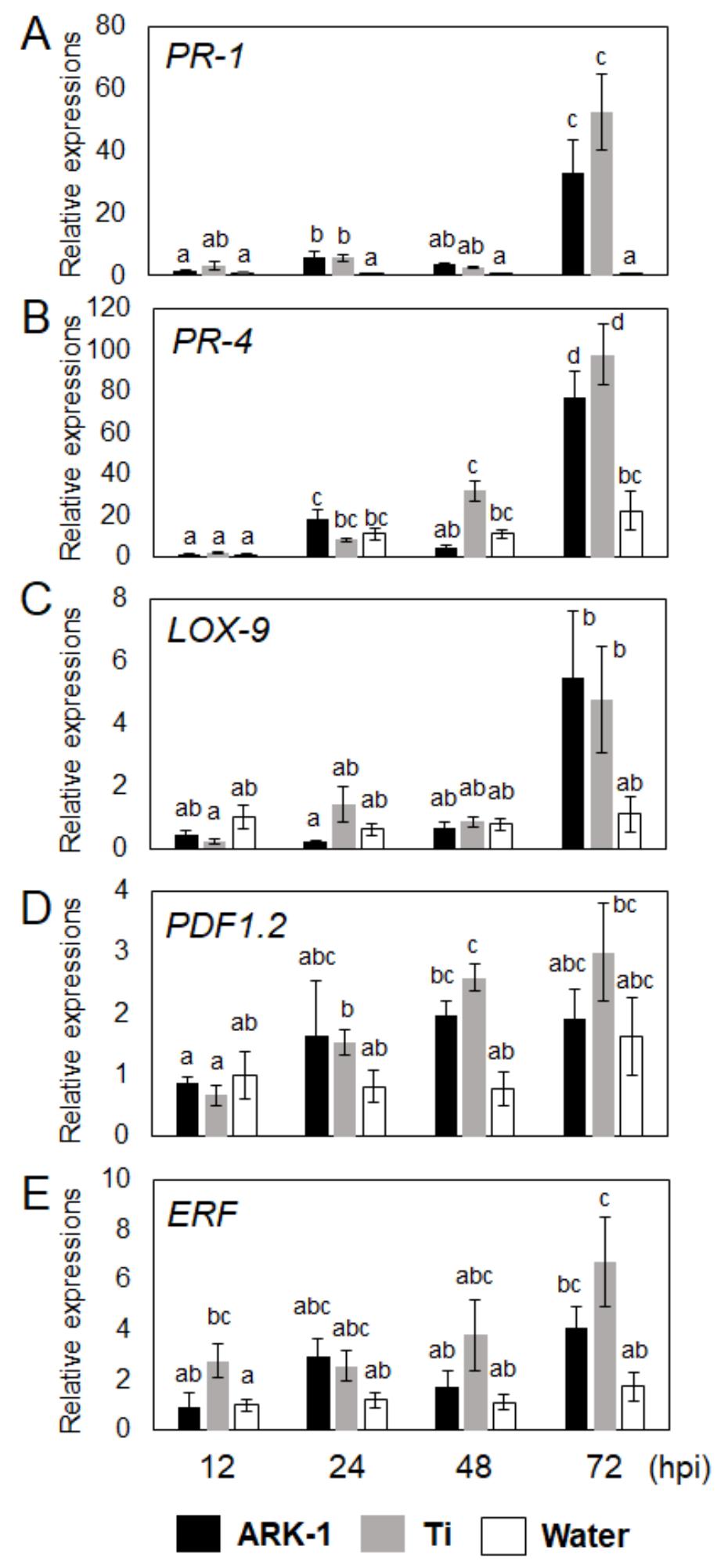

Figure 3

Induction of defense-related genes by ARK-1 and Ti during 3 days post-inoculation in grapevine seedlings. ARK-1, Ti, or sterile distilled water was inoculated into the middle position, and tissues $5 \mathrm{~mm}$ 
away from the inoculation sites were collected at the indicated time points. The relative expression rates of defense-related genes PR-1 (A), PR-4 (B), LOX-9 (C), PDF1.2 (D), and ERF (E) were measured using reverse transcriptase quantitative polymerase chain reaction (RT-qPCR) with specific primers. Data are means $\pm S E M$ of relative expression rates to each value with water at 12 hours post-inoculation (hpi), which had a value of $1.0(n=3)$. Different letters represent significant differences according to Tukey's HSD $(P \leq 0.05)$. The experiments were repeated 3 times with similar results.

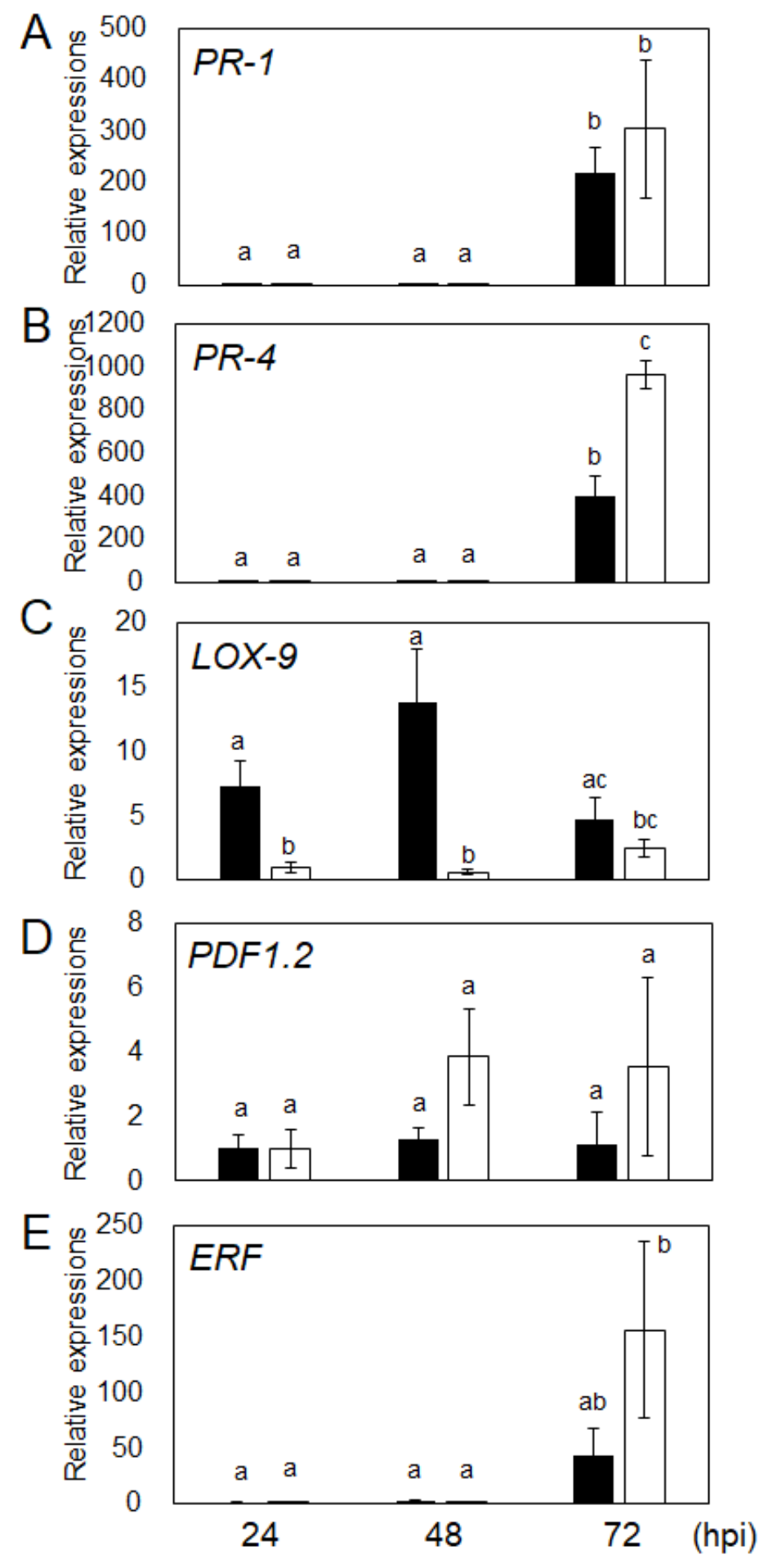

Ti 5 dpi of ARK-1 $\square$ Ti 5 dpi of water

Figure 4 
Priming effect of pre-inoculation of ARK-1 on the induction of defense-related genes induced by Ti. ARK-1 or sterile distilled water was inoculated into both upper and lower positions in grapevine seedlings, and $\mathrm{Ti}$ was inoculated into the middle position after 5 days. Tissues $5 \mathrm{~mm}$ away from the inoculation sites were collected at the indicated time points. The relative expressions of defense-related genes PR-1 (A), PR-4 (B), LOX-9 (C), PDF1.2 (D), and ERF (E) were measured using reverse transcriptase quantitative polymerase chain reaction (RT-qPCR) with specific primers. Data are means $\pm S E M$ of relative expression rates to each value with water at 12 hours post-inoculation (hpi), which had a value of $1.0(n=3)$. Different letters represent significant differences according to Tukey's HSD $(P \leq 0.05)$. The experiments were repeated 3 times with similar results. 

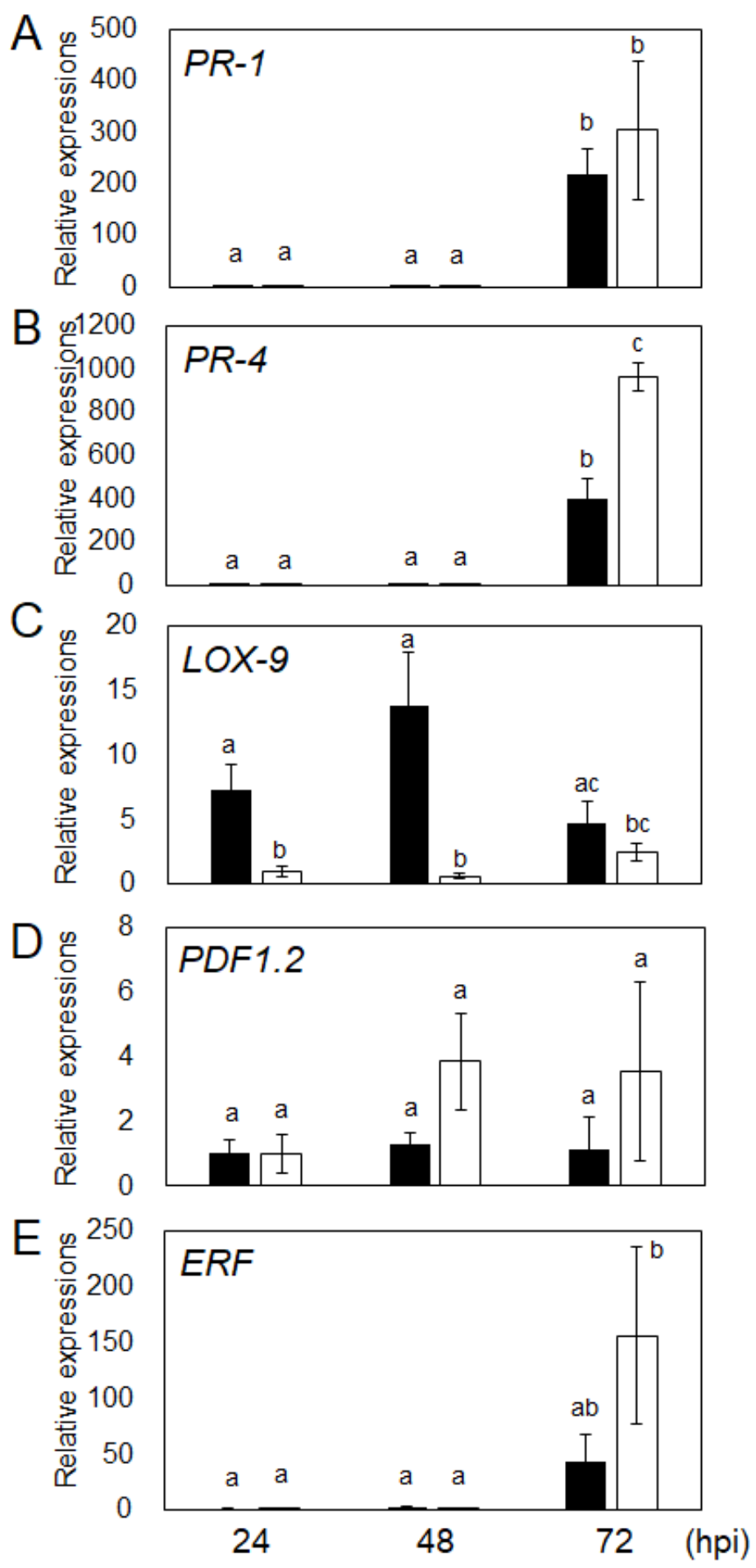

$\square$ Ti 5 dpi of ARK-1 $\square$ Ti 5 dpi of water

\section{Figure 4}

Priming effect of pre-inoculation of ARK-1 on the induction of defense-related genes induced by Ti. ARK-1 or sterile distilled water was inoculated into both upper and lower positions in grapevine seedlings, and $\mathrm{Ti}$ was inoculated into the middle position after 5 days. Tissues $5 \mathrm{~mm}$ away from the inoculation sites were collected at the indicated time points. The relative expressions of defense-related genes PR-1 (A), PR-4 (B), LOX-9 (C), PDF1.2 (D), and ERF (E) were measured using reverse transcriptase quantitative 
polymerase chain reaction (RT-qPCR) with specific primers. Data are means $\pm S E M$ of relative expression rates to each value with water at 12 hours post-inoculation (hpi), which had a value of $1.0(n=3)$. Different letters represent significant differences according to Tukey's HSD ( $P \leq 0.05)$. The experiments were repeated 3 times with similar results.

\section{Supplementary Files}

This is a list of supplementary files associated with this preprint. Click to download.

- KawaguchietalSupplimentalFigsandTables.pptx

- KawaguchietalSupplimentalFigsandTables.pptx 